Typeset by REVTEX 4 for JASA

\title{
Resonance modes in a 1D medium with two purely resistive boundaries: calculation methods, orthogonality and completeness
}

\author{
Jean Kergomard ${ }^{\text {a) }}$ and Vincent Debut \\ Laboratoire de Mécanique et d'Acoustique, CNRS UPR 7051, 31 Chemin \\ Joseph Aiguier, 13402 Marseille Cedex 20, France \\ Denis Matignon \\ Télécom Paris/Département TSI, CNRS UMR 5141 \\ 37-39, rue Dareau 75014 Paris, France
}

(Dated: November 13, 2018)

\begin{abstract}
Studying the problem of wave propagation in media with resistive boundaries can be made by searching for "resonance modes" or free oscillations regimes. In the present article, a simple case is investigated, which allows one to enlighten the respective interest of different, classical methods, some of them being rather delicate. This case is the $1 \mathrm{D}$ propagation in a homogeneous medium having two purely resistive terminations, the calculation of the Green function being done without any approximation using three methods. The first one is the straightforward use of the closed-form solution in the frequency domain and the residue calculus. Then the method of separation of variables (space and time) leads to a solution depending on the initial conditions. The question of the orthogonality and completeness of the complex-valued resonance modes is investigated, leading to the expression of a particular scalar product. The last method is the expansion in biorthogonal modes in the frequency domain, the modes having eigenfrequencies depending on the frequency. Results of the three methods generalize or/and correct some results already existing in the literature, and exhibit the particular difficulty of the treatment of the constant mode.
\end{abstract}

PACS numbers: $43.20 \mathrm{Ks}, 43.40 \mathrm{Cw}, 02.30 \mathrm{~Tb}, 02.30 \mathrm{Jr}$

\section{INTRODUCTION}

Studying the problem of wave propagation in media with resistive boundaries can be made by searching for "resonance modes" (see Ref.1), or free oscillations regimes. These modes can be non-orthogonal for the ordinary scalar product, entailing some difficulties depending on the mathematical treatment, made either in the time or frequency domain. Two classical methods exist for such a problem, and can be used either for a scalar, second order differential equation, or for a system of two equations of the first order. They have been especially used for the problem of a 1D medium with one resistive boundary, the other boundary condition being of Dirichlet type:

i) in the time domain, the use of time and space variable as separate variables leads directly to the basis of modes, but they are non-orthogonal for the most common product, and difficulties occur when searching for the coefficients depending for instance on initial conditions. Nevertheless, for a particular case, Oliveto and Santini ${ }^{2}$ and Guyader $^{3}$ have solved the problem, and Rideau ${ }^{4}$ using a system of equations of the first order,

a) Electronic address: kergomard@lma.cnrs-mrs.fr found a scalar product making the modes orthogonal (see also Refs.5-7), and gave the proof of completeness.

ii) in the frequency domain, the equations to be solved are ordinary differential equations with boundary conditions depending on frequency, but the use of orthogonal decomposition is possible. This leads to eigenmodes and eigenfrequencies depending on frequency. It is the case for the classical theory of room acoustics (see e.g. Morse and Ingard $^{8}$ ), using biorthogonality. To return to time domain in order to deduce the resonance modes is a rather delicate task, especially because of the calculation of the derivation of eigenfrequencies with respect to frequency. Biorthogonality has been used also for duct modes (see e.g. Ref.9) Another approach has been recently used by Trautmann and Rabenstein, ${ }^{10,11}$ using a system of first order equations (these authors treat the case of two resistive boundary conditions).

The present article is devoted to the study of the simple 1D case, when the two boundaries are resistive. One goal is to exhibit how the different methods articulate. We start by using the fact that a straightforward solution exists for the wave equation with source, by applying the residue calculus to the closed-form of the Fourier domain solution: as discussed by Levine, ${ }^{12}$ this closed-form solution, avoiding the sum of a series, is "relatively poorly, if not entirely, unknown to the general acoustics community". All calculations can be carried out analytically 
without any approximation, exhibiting the properties of the different methods (however many previous papers restrict their content to small impedance, or admittance, at one extremity, using perturbation methods). The case under study corresponds to one-dimensional propagation in a homogeneous medium bounded by two other semiinfinite media with different characteristic impedances, dissipation being therefore due to radiation at infinity. It is especially interesting because of its physical significance (it is probably the simplest radiation problem), and also because it realizes one of the possible transitions between Neumann and Dirichlet boundary conditions. Notice that in the context of optics and quantum mechanics, the problem has been studied including the outside media by Leung et al, ${ }^{13,14}$ the resonance modes being called quasinormal modes.

In section II, the equations to be solved are stated, with some possible physical interpretations. As a first step, the classical, closed-form solution of the Green function in the frequency domain is established (section III), with its inverse Fourier Transform, corresponding to the successive reflections (section IV). The second step is the residue calculus in order to determine the resonance modes (section $\mathrm{V}$, the basic result being given by Eqs. (26)). Then results are compared to those of the two aforementioned methods, i.e.: i) the method of separation of variables (section VI), which gives the result for given initial conditions (the corresponding results being Eqs. (37), (48) and (49)); in this section, the question of orthogonality and completeness of the modes is investigated. ii) the method of eigenmodes in the frequency domain (section VII). For the two methods, both second order scalar equation and first order system of two equations are used successively, with emphasis on the existence of a constant mode.

\section{STATEMENT OF THE PROBLEM, PHYSICAL INTERPRETATION}

The Green function $g\left(x, t \mid x_{0}, t_{0}\right)$ for the wave equation is solution of the following equation:

$$
\left[\partial_{x x}^{2}-c^{-2} \partial_{t t}^{2}\right] g(x, t)=-\delta\left(x-x_{0}\right) \delta\left(t-t_{0}\right)
$$

where $x$ and $x_{0}$ are the spatial coordinates of the receiver and source, respectively (or vice-versa), $t$ and $t_{0}$ the times of observation and excitation, respectively, $c$ the speed of sound. $\delta(x)$ is the Dirac function.

For sake of simplicity, $x_{0}$ and $t_{0}$ are considered to be fixed. Moreover in the whole paper, the choice of $t_{0}=0$ is made. For negative $t$, the function is zero, as well as its first derivative. The Green function satisfies the following boundary conditions:

$$
\begin{array}{r}
c \zeta \partial_{x} g(x, t)=\partial_{t} g(x, t) \quad \text { at } x=0, \\
c \zeta_{\ell} \partial_{x} g(x, t)=-\partial_{t} g(x, t) \quad \text { at } x=\ell
\end{array}
$$

where $\zeta=Z / \rho c, \rho$ is the density of the fluid, and $Z$ the impedance at $x=0$, which is assumed to be a

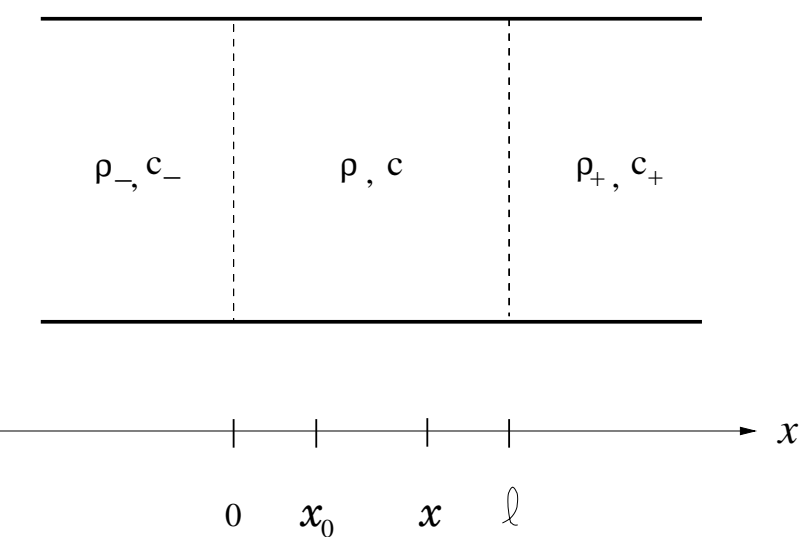

FIG. 1. 1D medium bounded with two other media.

real quantity, independent of the frequency. Similarly, $\zeta_{\ell}=Z_{\ell} / \rho c$, where $Z_{\ell}$ is the impedance at $x=\ell(\ell$ being positive).

An obvious physical interpretation for quantities $\zeta$ and $\zeta_{\ell}$ is the following: consider for $x<0$ and $x>\ell$ (see figure 1) two media with characteristic impedances $\rho_{-} c_{-}$ and $\rho_{+} c_{+}$, respectively. If the media are non dissipative, impedances are real, and can be larger or smaller than the impedance of the bounded medium, $\rho c$. Moreover, they are positive, because they correspond to waves outgoing from the bounded medium. Therefore this is the problem of planar pressure waves in a stratified medium, the direction of propagation being normal to the interfaces. A generalization to more complex stratified media would be possible, at least numerically. In this problem, the Green function corresponds to the acoustic pressure: of course, it has not the dimension of a pressure, but the solution for a "concrete" problem with source can be easily solved, as explained in standard textbooks, and discussed in a recent paper by Levine. ${ }^{12}$

Other problems correspond to the previous equations:

i) in an approximate way, ignoring higher order duct modes, the problem of planar waves in a rigid walled duct terminating in two semi-infinite ducts with different cross sections areas, the quantities $\zeta$ and $\zeta_{\ell}$ being the ratios of the areas. The approximation is good at low frequencies.

ii) the problem of a dissipative termination : the terminal impedances $Z$ and $Z_{\ell}$ can be the impedances of dissipative media (at low frequencies, a porous medium open to a large space can be an approximation of a pure resistance, due to viscous effects).

In all the previous problems, the quantities $\zeta$ and $\zeta_{\ell}$ are real and positive, the terminations being passive. For active terminations, they can be negative. An example is the beginning of self-sustained oscillations in musical instruments: a nonlinear excitator, like a reed for a clarinet, can be linearized as a pure resistance. When the main control parameter, i.e. the pressure in the mouth of the musician, increases, the resistance becomes negative, the static regime becomes unstable, and an oscillation starts as an increasing exponential (see e.g. Refs.15,16). 
Obviously analogous problems for mechanical vibrations or other wave fields are numerous.

\section{CLOSED-FORM SOLUTION FOR THE FOURIER TRANSFORM}

The Fourier Transform (FT) of $g(x, t)$ is denoted $G(x, \omega)$ (throughout the article, functions of time are written in small characters, and their FT are written in capital characters). It is equal to:

$$
\begin{array}{r}
G(x, \omega)=\int_{-\infty}^{+\infty} g(x, t) e^{-i \omega t} d t, \text { where } \\
g(x, t)=\frac{1}{2 \pi} \int_{-\infty}^{+\infty} G(x, \omega) e^{i \omega t} d \omega .
\end{array}
$$

The FT of Eqs. (1) to (3) are found to be:

$$
\begin{array}{r}
\left(\partial_{x x}^{2}+\omega^{2} / c^{2}\right) G(x, \omega)=-\delta\left(x-x_{0}\right), \\
c \zeta \partial_{x} G(x, \omega)=i \omega G(x, \omega) \text { at } x=0 ; \\
c \zeta_{\ell} \partial_{x} G(x, \omega)=-i \omega G(x, \omega) \text { at } x=\ell .
\end{array}
$$

While terminal impedances are independent of frequency, boundary conditions are frequency dependent. Nevertheless a classical, closed-form, solution is already known, which has been especially used in Ref.17. If $x \neq x_{0}$ solutions of Eq. (6) can be written as:

$$
\begin{array}{r}
G(x, \omega)=A^{-} \cosh [i \omega x / c+\eta] \text { if } x<x_{0} ; \\
G(x, \omega)=A^{+} \cosh \left[i \omega(\ell-x) / c+\eta_{\ell}\right] \text { if } x>x_{0} .
\end{array}
$$

For the boundary conditions, the following definitions are used:

$$
\begin{aligned}
\zeta & =\operatorname{coth} \eta ; r=e^{-2 \eta}=(\zeta-1) /(\zeta+1) \\
\zeta_{\ell} & =\operatorname{coth} \eta_{\ell} ; r_{\ell}=e^{-2 \eta_{\ell}}=\left(\zeta_{\ell}-1\right) /\left(\zeta_{\ell}+1\right),
\end{aligned}
$$

where $r$ and $r_{\ell}$ are the reflection coefficients. The quantity $\eta$ satisfies: $2 \eta=-(\ln |r|+i \arg (r))[2 \pi]$. Because $r$ is real, we choose the following definition:

$$
\eta=\eta_{r}+i \mu \pi / 2 ; \mu=0 \text { or } 1 .
$$

Two cases exist: i) if $|\zeta|>1, r>0, \mu=0$; ii) if $|\zeta|<$ $1, r<0, \mu=1$. Similar remark and definition can be applied to boundary $x=\ell$ :

$$
\eta_{\ell}=\eta_{\ell r}+i \mu_{\ell} \pi / 2 ; \mu_{\ell}=0 \text { or } 1 .
$$

The case $\zeta=1$ (semi-infinite tube or medium) corresponds to $\eta=\infty$ : it is discussed in the next sections. Except for the last one, most of the following calculations are valid for all cases. At $x=x_{0}$, writing the continuity of the function and the jump of its first derivative, the following result is obtained:

$$
G(x, \omega)=\frac{c}{i \omega} \frac{\cosh \left[\eta+i \omega x_{0} / c\right] \cosh \left[\eta_{\ell}+i \omega(\ell-x) / c\right]}{\sinh \left(i \omega \ell / c+\eta+\eta_{\ell}\right)}
$$

if $x \geq x_{0}$ and a similar result if $x \leq x_{0}$, by interchanging $x$ and $x_{0}$.

\section{SOLUTION IN THE TIME DOMAIN (SUCCESSIVE REFLECTIONS)}

Eq. (12) can be transformed in the time domain, leading to a solution corresponding to the successive reflections of the Green function in infinite space at the two boundaries. It will be the reference solution for the check of the validity of the modal expansion. The sinh function of the denominator can be written as

$$
\sinh \left(i \omega \ell / c+\eta+\eta_{\ell}\right)=\frac{1-e^{-2 \eta-2 \eta_{\ell}-2 i \omega \ell / c}}{2 e^{-\eta-\eta_{\ell}-i \omega \ell / c}}
$$

and, if the modulus of the exponential at the denominator is less than unity (this is discussed hereafter), as:

$$
\begin{array}{r}
\sinh ^{-1}\left(i \omega \ell / c+\eta+\eta_{\ell}\right)=2 e^{-\eta-\eta_{\ell}-i \omega \ell / c} \\
{\left[1+F(\omega)+F^{2}(\omega)+F^{3}(\omega)+\ldots\right] .}
\end{array}
$$

$F(\omega)=\exp \left(-2 \eta-2 \eta_{\ell}-2 i \omega \ell / c\right)$ is the function corresponding to a complete round trip of a wave in the tube, of duration $2 \ell / c$. Concerning the numerator of (12), it can be written: $\exp \left(+\eta+\eta_{\ell}+i \omega \ell / c\right) G_{p}(x, \omega) c / 4$, where:

$$
\begin{gathered}
G_{p}(x, \omega)=e^{-i \omega\left(x-x_{0}\right) / c}+r e^{-i \omega\left(x+x_{0}\right) / c} \\
+r_{\ell} e^{-i \omega\left(2 \ell-x-x_{0}\right) / c}+r r_{\ell} e^{-i \omega\left(2 \ell-x+x_{0}\right) / c} .
\end{gathered}
$$

Therefore the Green function is:

$$
G(x, \omega)=\frac{c}{2 i \omega} G_{p}(x, \omega)\left[1+F(\omega)+F^{2}(\omega)+\ldots\right] .
$$

The factor $G_{p}(x, \omega) / i \omega$ corresponds to the four "primary" waves arriving during the first cycle of duration $2 \ell / c$, and this packet is simply reproduced at times $2 \ell / c, 4 \ell / c, 6 \ell / c$, etc... (see for a detailed explanation e.g. Kergomard $\left.{ }^{15}\right)$. The inverse FT of the function $G_{p}(x, \omega) / i \omega$, denoted $h_{p}(x, t)$, is obtained by taking into account the zero condition for negative times. The result is found to be, whatever the sign of $\left(x-x_{0}\right)$ :

$$
\begin{gathered}
h_{p}(x, t)=H\left[t-\left|x-x_{0}\right| / c\right]+r H\left[t-\left(x+x_{0}\right) / c\right]+ \\
r H\left[t-\left(2 \ell-x-x_{0}\right) / c\right]+r r_{\ell} H\left[t-\left(2 \ell-\left|x-x_{0}\right|\right) / \emptyset 16\right)
\end{gathered}
$$

where $H(t)$ is the step function. Finally

$$
\begin{array}{r}
g(x, t)=\frac{c}{2} h_{p}(x, t) *[\delta(t)+f(t)+(f * f)(t)+\ldots] \\
f(t)=r r_{\ell} \delta(t-2 \ell / c) \\
.
\end{array}
$$

Condition of validity of expansion (13) is $\left|r r_{\ell}\right|<1$. We notice that if $\zeta$ is real and positive, $|r|<1$, and similarly for $\zeta_{\ell}$. Therefore the condition is satisfied when the two boundaries are dissipative, or, more precisely, if the combination of the two reflections is dissipative. The case $\left|r r_{\ell}\right|>1$ will be discussed in section VC. Other comments can be made:

- the article is limited to purely resistive boundaries, but Eqs. (16) and (18) can be generalized to various 
boundary conditions defined by a reflection coefficient, $R(\omega)$. This is done by replacing the products like $r H(t)$ by the convolution product $(r * H)(t)$, where $r(t)$ is the inverse FT of $R(\omega)$.

- for the case under study, we notice that the convolution product of $n$ times function $f(t)$ is $\left(r r_{\ell}\right)^{n} \delta(t-2 n \ell / c)$.

- if $\zeta$ (respectively $\zeta_{\ell}$ ) is unity, the reflection coefficient $r$ (respectively $r_{\ell}$ ) vanishes, as well as $f(t)$ : the first term of the Green function is the Green function of an infinite medium, the first two terms correspond to a semi-infinite medium, etc... As it will be seen in the next section, no modes can be found for these cases, because no reflections exist, either $\eta$ or $\eta_{\ell}$ tending to infinity.

- finally, multiplying both members of Eq. (15) by the factor $[1-F(\omega)]$, and taking the inverse FT, it appears that a closed-form exists in the time domain, which is the basis for the study of the Helmholtz motion of bowed string instruments (see e.g. Woodhouse ${ }^{18}$ ). It is a recurrence relationship:

$$
\partial_{t} g(x, t)-r r_{\ell} \partial_{t} g(x, t-2 \ell / c)=g_{p}(x, t) c / 2 .
$$

\section{EXPANSION IN RESONANCE MODES USING THE INVERSE FT}

Putting expression (12) of the frequency domain in Eq. (5) leads to the modal expansion of the time domain expression. The tool is the residue calculus. If all poles of expression (12) are simple and located on or above the real axis, the following equation can be used:

$$
g(x, t)=i \Sigma \text { if } t>0 \text { and } 0 \text { if } t<0,
$$

where $\Sigma$ is the sum of the residues of $G(x, \omega) \exp (i \omega t)$ (see e.g. Morse and $\operatorname{Ingard}^{8} \mathrm{p} 17$, changing $i$ to $-i$ ).

\section{A. Calculation of the poles}

Zeros of function sinh satisfy:

$$
i \omega_{n}=\left[-\eta-\eta_{\ell}+i n \pi\right] c / \ell,
$$

where $n$ is an integer. In order for the poles to be above the real axis, the condition is $\eta_{r}+\eta_{\ell r}>0$. It is equivalent to the condition previously obtained for the successive reflections expansion: $\left|r r_{\ell}\right|<1$. Using definition (10), Eq. (20) is rewritten as:

$$
\omega_{n}=\left[n-\left(\mu+\mu_{\ell}\right) / 2\right] \pi c / \ell+i\left(\eta_{r}+\eta_{\ell r}\right) c / \ell .
$$

As already remarked by several authors, the imaginary part of the complex frequency is independent of $n$, and the real part is independent of the dissipation. Depending on the values of $\zeta$ and $\zeta_{\ell}$, different cases must be distinguished:

i) if $|\zeta|>1$ and $\left|\zeta_{\ell}\right|>1$ (real $\eta$ and $\eta_{\ell}$ ): the real part of the frequency corresponds to the values for pure Neumann conditions (infinite $\zeta$ and $\zeta_{\ell}$ ). ii) if $|\zeta|>1$ and $\left|\zeta_{\ell}\right|<1$ (mixed case with either complex $\eta$ or complex $\eta_{\ell}$ : either $\mu$ or $\mu_{\ell}$ is unity): the real part corresponds to a problem with different conditions (Neumann and Dirichlet) at $x=0$ at $x=\ell$. The real part of eigenfrequencies is an odd harmonic of $c / 4 \ell$.

iii) if $|\zeta|<1$ and $\left|\zeta_{\ell}\right|<1$ (complex $\eta$ and $\eta_{\ell}: \quad \mu=$ $\left.\mu_{\ell}=1\right)$ : the real part corresponds to the values for pure Dirichlet conditions (zero $\zeta$ and $\zeta_{\ell}$ ).

Except for case ii), a purely imaginary eigenfrequency exists for $n=\left(\mu+\mu_{\ell}\right) / 2$.

\section{B. Calculation of the residues}

In all cases, the Taylor expansion of the function sinh in Eq. (12) at the first order of the quantity $\left(\omega-\omega_{n}\right)$ can be determined. The result is:

$$
\sinh \left[i \omega \ell / c+\eta+\eta_{\ell}\right] \simeq i(-1)^{n}\left(\omega-\omega_{n}\right) \ell / c .
$$

We get for $\omega$ close to the pole $\omega_{n}$ :

$$
\begin{array}{r}
G(x, \omega)=-\frac{c^{2}}{\omega_{n} \ell} \frac{f_{n}(x) f_{n}\left(x_{0}\right)}{\left(\omega-\omega_{n}\right)} \\
f_{n}(x)=\cosh \left(\eta+i \omega_{n} x / c\right)
\end{array}
$$

or $f_{n}(x)=(-1)^{n} \cosh \left(i \omega_{n}(\ell-x) / c+\eta_{\ell}\right)$. The residue corresponding to the pole $\omega=0$, remains to be calculated. For small $\omega$,

$$
G(x, \omega)=\frac{c}{i \omega} \frac{\cosh \eta \cosh \eta_{\ell}}{\sinh \left(\eta+\eta_{\ell}\right)}=\frac{c}{i \omega} \frac{1}{\zeta^{-1}+\zeta_{\ell}^{-1}} .
$$

Using Eq. (19), the inverse FT of $G(x, \omega)$ is obtained:

$$
g(x, t)=H(t) \frac{c^{2}}{\ell} \sum_{n} \frac{f_{n}(x) f_{n}\left(x_{0}\right)}{i \omega_{n}} e^{i \omega_{n} t}+\frac{c H(t)}{\zeta^{-1}+\zeta_{\ell}^{-1}} .
$$

Some comments can be made:

- the formula is valid for all aforementioned cases;

- the mode shapes $f_{n}(x)$ are complex-valued functions of the space variable, meaning that the shape is varying with time (for a discussion on complex modes, see e.g.Ref.19). The question of their orthogonality will be discussed in section VI. Notice that functions $f_{n}(x)$ do not fulfill the same boundary conditions than $G(x, \omega)$ : the boundary conditions are (7) and (8), but where $\omega$ is replaced by $\omega_{n}$;

- the last term in Eq. (26) is a constant mode. If one of the impedances $\zeta$ or $\zeta_{\ell}$ is zero, it disappears, as it is intuitive, in order to satisfy a Dirichlet condition. It is a trivial solution of the wave equation and the boundary conditions, and can be compared to the DC component of a periodic signal. When both $\zeta$ and $\zeta_{\ell}$ tend to infinity, the boundaries tend to Neumann boundaries, and the combination of the non oscillatory mode of frequency $\omega_{0}$ and the constant mode results in a uniform (i.e. constant 
in space) mode which increases linearly with time. The calculation is done as follows: if $\eta$ and $\eta_{\ell}$ tend to zero, $\omega_{0}$ tends to zero, and the factor $\exp \left(i \omega_{0} t\right)$ can be written as: $1+i \omega_{0} t$. The zeroth order term is equal to the opposite of the constant mode, and only the linear term remains. The result is $H(t) t c^{2} / \ell$, and its FT is $-c^{2} / \ell \omega^{2}$. This mode is the classical uniform mode existing for instance in $3 \mathrm{D}$ cavities with rigid walls: curiously it exists in the standard textbooks (see e.g. Ref.8, page 571), but the time domain expression is not given. This mode is similar to the well known planar guided mode, existing in ducts with rigid walls, whatever the geometrical shape.

- the imaginary part of the complex frequencies being independent of $n$, the decay is identical for all non constant modes;

- for the above-considered case i), we notice that $\omega_{-n}=$ $-\omega_{n}^{*}$ and $f_{-n}(x)=f_{n}^{*}(x)$, and, more generally:

$$
i \omega_{\nu}=\left(i \omega_{n}\right)^{*} ; f_{\nu}(x)=(-1)^{\mu} f_{n}^{*}(x),
$$

where $\nu=-n+\mu+\mu_{\ell}$ is an integer.

As a consequence, the solution $g(x, t)$ is real. It could be possible to transform the sum by adding the two oscillating terms corresponding to $n$ and $\nu$, when $n \neq \nu$, as it is usually done for non dissipative boundaries. Nevertheless it appears that the formulas become intricate.

- Eqs. (23) and (25) lead directly to another form of the FT of result (26), written as a series:

$$
G(x, \omega)=-\frac{c^{2}}{\ell} \sum_{n} \frac{f_{n}(x) f_{n}\left(x_{0}\right)}{\omega_{n}\left(\omega-\omega_{n}\right)}+\frac{c}{i \omega} \frac{1}{\zeta^{-1}+\zeta_{\ell}^{-1}}
$$

An example of comparison of the successive reflections method and modal expansion is shown in figure 2(a), for conditions close to Neumann and Dirichlet. We notice that it is satisfactory. The Gibbs phenomenon appears, because of the truncated series of modes, ensuring the correct accordance between the two methods. Moreover this accordance confirms the existence of the constant mode. Decreasing of the maxima is due to the dissipation at the boundaries: for pure Neumann and Dirichlet conditions, the shape would be similar, but perfectly periodical.

\section{The case of active boundaries}

What happens when the combination of boundaries is active, i.e. when $\left|r r_{\ell}\right|>1$, or $\eta_{r}+\eta_{\ell r}<0$ (at least one of the impedances $\zeta$ or $\zeta_{\ell}$ is negative)? It is possible to prove that Eqs. (17) and (26) remain valid for active boundary conditions, as explained hereafter. The real part of $i \omega_{n}$ being independent of $n$, this suggests to use a new function $\widetilde{g}(x, t)=g(x, t) \exp (-\widetilde{\eta} t)$, where $\widetilde{\eta}>-\eta_{r}-$ $\eta_{\ell r}>0$, which can be substituted in the initial problem in order for the poles to be located again on or above the real axis. Eq. (1) becomes

$$
\partial_{x x}^{2} \widetilde{g}(x, t)-c^{-2}\left[\partial_{t}+\widetilde{\eta}\right]^{2} \widetilde{g}(x, t)=-\delta\left(x-x_{0}\right) e^{-\widetilde{\eta} t} \delta(t)
$$

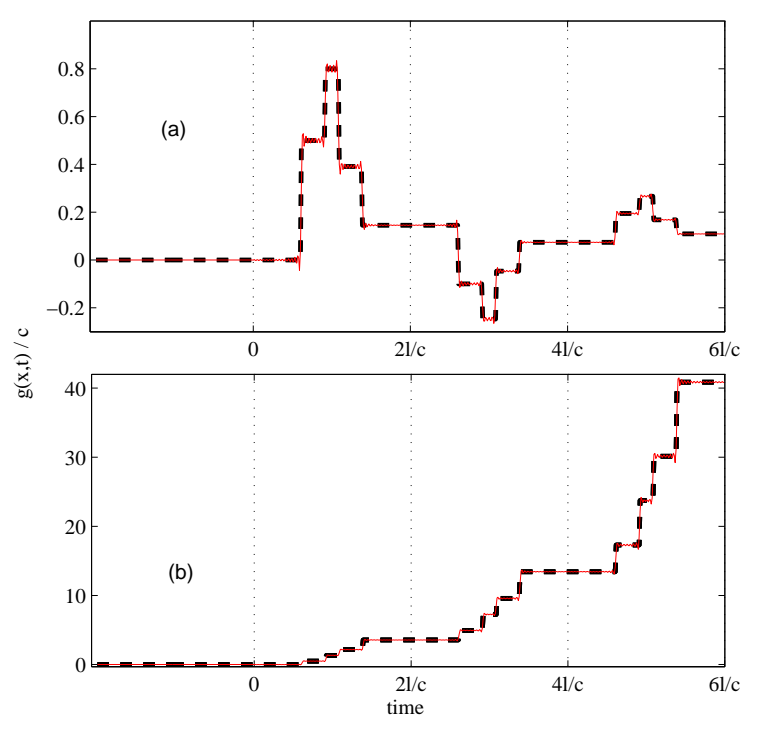

FIG. 2. Normalized Green function as a function of time: comparison between the successive reflections method (dotted line) and modal expansion (solid line, 102 modes, i.e. maximum $n=50$ ). Locations of the source and receiver are $x_{0} / \ell=0.15$ and $x / \ell=0.76$, respectively.

(a) passive boundaries $\zeta=4$. ; $\zeta_{\ell}=0.1$ (constant mode= 0.097). (b) active boundary $\zeta=\zeta_{\ell}=-4$. (constant mode $=$ $-2)$.

and similarly for Eqs. (2) and (3). It is equivalent to use an appropriate Laplace Transform. Going in the frequency domain leads to Eqs. (6 to 8), where $G(x, \omega)$ is replaced by $\widetilde{G}(x, \omega)$ and $i \omega$ by $(i \omega+\widetilde{\eta})$, and a similar result for Eq. (12). The analysis of both successive reflections and poles and residues leads to the result $\widetilde{g}(x, t)=g(x, t) \exp (-\tilde{\eta} t)$, where $g(x, t)$ is given by Eqs. (17) and (26), respectively, and the proof is achieved. We do not repeat here the complete procedure. We notice that for the case $\eta_{r}+\eta_{\ell r}=0$, one boundary is active and the other one is passive: eigenfrequencies $\omega_{n}$ are real while modes are complex. Figure 2(b) shows an example of result.

\section{METHOD OF SEPARATION OF VARIABLES}

\section{A. Second order homogeneous equation with initial conditions}

\section{Derivation of the modes}

Oliveto and Santini, ${ }^{2}$ and Guyader $^{3}$ have treated a particular case of the problem (zero $\zeta$, large $\zeta_{\ell}$ ) using the method of separation of variables. He gets non orthogonal modes for the common scalar product $\int_{0}^{\ell} f_{n}(x) f_{m}(x) d x$. We will see that the method is valid whatever the values of the two boundary conditions, and how the derivation can be simplified. 
We are searching for solutions $p(x, t)$ of homogeneous equation (1) (without second member), with boundary conditions (2) and (3), and with given initial conditions. Assuming that the general solution is a superposition of solutions with separate variables, the solutions with separate variables are written in the following form:

$$
\begin{array}{r}
p(x, t)=f(x) h(t) ; \\
h(t)=B^{+} e^{i \omega t}+B^{-} e^{-i \omega t} ; \\
f(x)=\cosh (i \omega x / c+\varphi) .
\end{array}
$$

Decomposition (30) differs from the ordinary FT, because a priori $\omega$ is a complex quantity, depending on the boundary conditions. Considering first the solution $B^{+} e^{i \omega t}$, this leads to :

$$
\begin{array}{r}
\zeta \omega \sinh \varphi=\omega \cosh \varphi \\
\omega \zeta_{\ell} \sinh (i \omega \ell / c+\varphi)=-\omega \cosh (i \omega \ell / c+\varphi) .
\end{array}
$$

$\omega=0$ is a solution, corresponding to the constant mode. The other modes are given by Eq. (33): $\sinh (\varphi-$ $\eta)=0$, thus:

$$
f(x)=\cosh (i \omega x / c+\eta) .
$$

Actually there is a sign \pm in the right-hand side member of Eq. ( 35 ), but it is without importance, because it can be included in the coefficient $B^{+}$of the solution. The eigenvalues equation is deduced from Eqs. (33) and (34), as follows:

$$
\sinh \left(i \omega \ell / c+\eta+\eta_{\ell}\right)=0
$$

the solutions being given by (20). The solution in time $B^{-} e^{-i \omega t}$ does not lead to new solutions for $f(x)$, therefore, assuming the solutions form a basis of solutions (this is discussed in section VIB), the general solution of a problem with initial conditions can be written as:

$$
p(x, t)=\sum_{n} A_{n} f_{n}(x) e^{i \omega_{n} t}+A,
$$

where $\omega_{n}$ and $f_{n}(x)$ are given by Eqs. (20) and (24), respectively, and the coefficients $A_{n}$ and $A$ depend on the initial conditions, and can be determined using the orthogonality relation of the modes. $A$ is the coefficient of the constant mode.

\section{Orthogonality relationship between the modes: first approach}

In order to derive an orthogonality relationship between the modes the common product is first calculated:

$$
\Lambda_{n m}=\int_{0}^{\ell} f_{n}(x) f_{m}(x) d x .
$$

Because $f_{n}(x)=(-1)^{\mu} f_{\nu}^{*}(x)$, the calculation of the quantities defined in Eq. (38) for all values of the index $n$ is equivalent to the calculation of the quantities defined when replacing $f_{m}(x)$ by its conjugate. Writing

$$
\begin{aligned}
& \int_{0}^{\ell}\left[f_{n}(x) \frac{d^{2} f_{m}(x)}{d x^{2}}-f_{m}(x) \frac{d^{2} f_{n}(x)}{d x^{2}}\right] d x= \\
& {\left[f_{n}(x) \frac{d f_{m}(x)}{d x}-f_{m}(x) \frac{d^{2} f_{n}(x)}{d x^{2}}\right]_{0}^{\ell}}
\end{aligned}
$$

and using Eq. (24), the following result is obtained:

$\frac{\left(\omega_{m}^{2}-\omega_{n}^{2}\right)}{c^{2}} \Lambda_{n m}=i\left(\omega_{m}-\omega_{n}\right)\left[\frac{f_{n}(0) f_{m}(0)}{\zeta}+\frac{f_{n}(\ell) f_{m}(\ell)}{\zeta_{\ell}}\right]$.

For $\omega_{m} \neq \omega_{n}$, because $\omega_{m}+\omega_{n} \neq 0$, the expression of $\Lambda_{n m}$ is deduced. For $\omega_{m}=\omega_{n}$, the calculation is straightforward. The general formula is found to be:

$$
\Lambda_{n m}=\frac{c i}{\omega_{m}+\omega_{n}}\left[\frac{f_{n}(0) f_{m}(0)}{\zeta}+\frac{f_{n}(\ell) f_{m}(\ell)}{\zeta_{\ell}}\right]+\frac{1}{2} \ell \delta_{n m}
$$

where $\delta_{n m}$ is the Kronecker symbol, or:

$$
\Lambda_{n m}=-\frac{c}{2} \frac{\sinh 2 \eta+(-1)^{n+m} \sinh 2 \eta_{\ell}}{i\left(\omega_{m}+\omega_{n}\right)}+\frac{1}{2} \ell \delta_{n m} .
$$

Modes are found to be non orthogonal for the product defined by (38), but, as shown by Guyader, ${ }^{3}$ it is possible to solve the problem from the knowledge of initial conditions. When dissipation tends to zero $\left(\eta_{r}\right.$ and $\eta_{\ell r}$ tend to zero), the first term does not vanish, tending to $(-1)^{\mu} \frac{1}{2} \ell \delta_{n, \nu}$. This is due to the choice of considering separately the modes $\omega_{n}$ and $\omega_{\nu}$.

Otherwise formula (39) remains valid when one of the modes is the constant mode $f(x)=1$, and the other one a non constant mode:

$$
\begin{array}{r}
\Lambda_{n}=\int_{0}^{\ell} f_{n}(x) d x=-\frac{c}{i \omega_{n}}\left[\frac{f_{n}(0)}{\zeta}+\frac{f_{n}(\ell)}{\zeta_{\ell}}\right] \\
=-\frac{c}{i \omega_{n}}\left(\sinh \eta+(-1)^{n} \sinh \eta_{\ell}\right) .
\end{array}
$$

Finally the product of the constant mode by itself is $\ell$.

\section{Solution with respect to initial conditions}

According to Eq. (37), the initial conditions are:

$$
\begin{aligned}
p(x, 0) & =\sum_{n} A_{n} \cosh \left(i \omega_{n} x / c+\eta\right)+A \\
\partial_{t} p(x, 0) & =\sum_{n} A_{n} i \omega_{n} \cosh \left(i \omega_{n} x / c+\eta\right) .
\end{aligned}
$$

Using Eq. (40) for a non constant mode $m$, the following results are obtained:

$$
\begin{gathered}
\int_{0}^{\ell} p(x, 0) f_{m}(x) d x=\sum_{n} A_{n} \Lambda_{n m}+A \Lambda_{m} ; \\
\int_{0}^{\ell} \partial_{t} p(x, 0) f_{m}(x) d x=\sum_{n} A_{n} i \omega_{n} \Lambda_{n m} .
\end{gathered}
$$


Multiplying Eq. (44) by $i \omega_{m}$, then adding Eq. (45), leads to:

$$
\begin{aligned}
& \int_{0}^{\ell}\left[i \omega_{m} p(x, 0)+\partial_{t} p(x, 0)\right] f_{m}(x) d x \\
& =i \sum_{n} A_{n}\left(\omega_{m}+\omega_{n}\right) \Lambda_{n m}+i A \omega_{m} \Lambda_{m} \\
& =-c \widehat{\sum_{n}} A_{n}\left[\frac{f_{n}(0) f_{m}(0)}{\zeta}+\frac{f_{n}(\ell) f_{m}(\ell)}{\zeta_{\ell}}\right]+i A_{m} \ell \omega_{m}(46) \\
& =-c\left[\frac{f_{m}(0) p(0,0)}{\zeta}+\frac{f_{m}(\ell) p(\ell, 0)}{\zeta_{\ell}}\right]+i A_{m} \ell \omega_{m}
\end{aligned}
$$

Notation $\widehat{\sum}$ for the series in Eq. (46) indicates that it involves the constant mode. As noticed by Guyader, ${ }^{3}$ this series is related to the initial conditions at the two ends $x=0$ and $x=\ell$. Thus for a non constant mode:

$$
\begin{aligned}
A_{n} \ell i \omega_{n}= & \int_{0}^{\ell}\left[i \omega_{n} p(x, 0)+\partial_{t} p(x, 0)\right] f_{n}(x) d x \\
& +c p(0,0) \sinh \eta+c p(\ell, 0) \varepsilon_{n} \sinh \eta_{\ell} .
\end{aligned}
$$

The following property is deduced from Eq. (27): $A_{\nu} f_{\nu}(x)=A_{n}^{*} f_{n}^{*}(x)$, thus $p(x, t)$ is real. Calculating $\int_{0}^{\ell} \partial_{t} p(x, 0) d x$, we similarly get coefficient $A$ :

$$
A=\frac{c^{-1} \int_{0}^{\ell} \partial_{t} p(x, 0) d x+p(0,0) \tanh \eta+p(\ell, 0) \tanh \eta_{\ell}}{\tanh \eta+\tanh \eta_{\ell}}
$$

What is the condition for which this coefficient vanishes? If for instance at $x=0, \zeta$ is zero, $\eta$ is infinite, and, according to the boundary condition, $p(0,0)$ vanishes, thus $A$ vanishes too. This confirms the remark concerning result (26).

Using the initial conditions for the Green function found in (17), it is possible to check result (26), but this will be done hereafter using the equation with source.

\section{B. First order system of equations, orthogonality and completeness of the modes}

\section{Introduction}

In this section we will prove that the modes form a Riesz basis in the space of solutions of a closely related problem, and give the expression of a scalar product making the modes orthogonal. As an introduction we show that a modified scalar product leads to the orthogonality of modes, except the constant one. For vibrating systems, the product defined by (38) corresponds to the product with respect to the mass, a complement being the calculation of the product related to the stiffness (see e.g. Meirovitch $^{20}$ ):

$$
\Lambda_{n m}^{\prime}=\int_{0}^{\ell} \frac{d}{d x} f_{n}(x) \frac{d}{d x} f_{m}(x) d x .
$$

By integrating by parts, and using Eq. (39), this product, for $n \neq m$, is found to be equal to:

$$
\Lambda_{n m}^{\prime}=\left[f_{n}(x) d_{x} f_{m}(x)\right]_{0}^{\ell}+\frac{\omega_{m}^{2}}{c^{2}} \Lambda_{n m}=-\frac{\omega_{n} \omega_{m}}{c^{2}} \Lambda_{n m} .
$$

Therefore the modes become orthogonal if we define a new product, as follows:

$$
\int_{0}^{\ell}\left[\partial_{x} p_{n} \partial_{x} p_{m}-\frac{1}{c^{2}} \partial_{t} p_{n} \partial_{t} p_{m}\right]_{t=0} d x=\delta_{n m} \ell \omega_{n}^{2} / c^{2},
$$

where $p_{n}=p_{n}(x, t)=f_{n}(x) \exp \left(i \omega_{n} t\right)$ and similarly for index $m$. We remark that the modes $p_{n}$ and $p_{\nu}=$ $(-1)^{\mu} p_{n}^{*}$ are orthogonal for this product. For the calculation of the solution from initial conditions, using Eq. (37) at $t=0$, the following result is obtained:

$\int_{0}^{\ell}\left[\frac{d}{d x} f_{n}(x) \partial_{x} p(x, 0)-\frac{i \omega_{n}}{c^{2}} f_{n}(x) \partial_{t} p(x, 0)\right] d x=A_{n} \frac{\omega_{n}^{2}}{c^{2}} \ell$.

As a consequence, the initial conditions need to be written by using the derivatives of the function $p(x, t)$ with respect to abscissa and time, respectively. Result (48) can be checked by integrating by parts the first term of the integral. Nevertheless, the product (50) is not useful for the constant mode, and the first method needs to be used (see subsection VI A 3). Moreover this derivation does not prove that the product is a scalar product, and that the modes form a basis of the space of solutions of the problem. This will be done hereafter.

\section{Riesz basis of the modes}

Several works have been done by mathematicians concerning spectral operators when boundary conditions are not simple conditions like Neumann or Dirichlet conditions. We quote the work by Russell, ${ }^{21}$ Majda, ${ }^{22}$ Lagnese,${ }^{23}$ Banks et al,${ }^{24}$ Darmawijoyo and Van Horssen, ${ }^{7}$ Cox and Zuazua. ${ }^{6}$ Rideau ${ }^{4}$ has treated the 1D case with a (unique) resistive termination, giving explicitly a scalar product (see also Ref.5). We generalize his calculation using a similar method, by considering the wave equation with source in the following form:

$$
\partial_{t} \boldsymbol{\psi}(x, t)=\boldsymbol{A} \boldsymbol{\psi}(x, t)+\boldsymbol{\phi}_{s}(x, t),
$$

where $\boldsymbol{\psi}(x, t)=(p, v)^{T}, p$ and $v /(\rho c)$ being the acoustic pressure and velocity, respectively. Operator $\boldsymbol{A}$ is:

$$
\boldsymbol{A}=\left(\begin{array}{cc}
0 & -c \partial_{x} \\
-c \partial_{x} & 0
\end{array}\right)
$$

and boundary conditions are written as:

$$
p(0, t)=-\zeta v(0, t) \text { and } p(\ell, t)=\zeta_{\ell} v(\ell, t) \quad \forall t .
$$

The family of eigenelements of $\boldsymbol{A}$ are found to satisfy:

$$
\lambda_{n} p_{n}(x)=-c \partial_{x} v_{n}(x) ; \lambda_{n} v_{n}(x)=-c \partial_{x} p_{n}(x),
$$


thus

$$
\begin{aligned}
\left(\begin{array}{c}
p_{n}(x) \\
v_{n}(x)
\end{array}\right) & =\left(\begin{array}{c}
\cosh \left(\lambda_{n} x / c+\eta\right) \\
-\sinh \left(\lambda_{n} x / c+\eta\right)
\end{array}\right) \\
\lambda_{n} & =\left(-\eta-\eta_{\ell}+i n \pi\right) c / \ell=i \omega_{n}
\end{aligned}
$$

(see Eq. $(20)) \cdot p_{n}(x)=f_{n}(x)$ and $\lambda_{n}$ are identical to the eigenfunctions and eigenvalues found before. Nevertheless the constant mode is eliminated (except for the very particular case $\eta=-\eta_{\ell}$ ), because the boundary conditions are slightly different: Eqs. (2) and (3) are obtained by deriving Eqs. (54) with respect to $t$. In Eq. (56) the argument of the hyperbolic functions can be written as:

$$
\begin{aligned}
\lambda_{n} x / c+\eta & =\alpha(x)+i \beta_{n}(x) ; \\
\alpha(x) & =-\eta_{\ell r} x / \ell+\eta_{r}(1-x / \ell) ; \\
\beta_{n}(x) & \left.=\pi\left[-\mu_{\ell} x / \ell+\mu(1-x / \ell)\right] / 2+n \pi x / \ell 60\right)
\end{aligned}
$$

(see definitions (10) and (11)). Denoting $\boldsymbol{\psi}_{n}^{\alpha}(x)=$ $\left(p_{n}(x), v_{n}(x)\right)^{T}$, we show in Appendix A that the family of elements $\boldsymbol{\psi}_{n}^{\alpha}(x)$ is a Riesz basis, i.e. a complete basis of elements, which become orthogonal for the following scalar product:

$$
<\boldsymbol{\psi}, \boldsymbol{\varphi}>_{H}^{\alpha}=\int_{0}^{\ell} \boldsymbol{\varphi}^{T *} \boldsymbol{G}_{2 \alpha}(x) \boldsymbol{\psi} d x
$$

$$
\text { where } \boldsymbol{G}_{\alpha}(x)=\left(\begin{array}{cc}
\cosh \alpha(x) & \sinh \alpha(x) \\
\sinh \alpha(x) & \cosh \alpha(x)
\end{array}\right) .
$$

For a given vector $\boldsymbol{\psi}=(p, v)^{T}$, the value of the scalar product with eigenvector $\boldsymbol{\psi}_{n}^{\alpha}$ is found to be:

$$
<\boldsymbol{\psi}, \boldsymbol{\psi}_{n}^{\alpha}>_{H}^{\alpha}=\int_{0}^{\ell}\left[p(x, t) p_{n}(x)-v(x, t) v_{n}(x)\right] d x .
$$

This is in accordance with the product (50). A direct application of this result is the solution of Eq. (52) with initial conditions $\boldsymbol{\psi}(x, 0)=(p(x, 0), v(x, 0))^{T}$. The modal decomposition is uniquely determined as $\boldsymbol{\psi}(x, t)=\sum_{n} h_{n}(t) \boldsymbol{\psi}_{n}^{\alpha}(x)$ in the energy space $H$, and leads to the following family of decoupled ordinary differential equations:

$$
\begin{aligned}
\ell\left[\partial_{t} h_{n}-\lambda_{n} h_{n}\right] & =<\phi_{s}(x, t), \boldsymbol{\psi}_{n}^{\alpha}>_{H}^{\alpha} ; \\
h_{n}(0) & =<\boldsymbol{\psi}(x, 0), \boldsymbol{\psi}_{n}^{\alpha}>_{H}^{\alpha}
\end{aligned}
$$

This result can be first applied to the calculation done in section VIA. In order to find a solution $\chi(x, t)$ of the second order equation without source, we denote $\boldsymbol{\psi}(x, t)=\left(\partial_{t} \chi(x, t),-c \partial_{x} \chi(x, t)\right)^{T}$, and obtain by integrating $\boldsymbol{\psi}(x, t)$ with respect to $t$ :

$$
\chi(x, 0)=\sum_{n} \lambda_{n}^{-1} h_{n}(0) p_{n}(x)+A .
$$

Using Eqs. (65), (63), and replacing $\chi(x, t)$ by $p(x, t)$, $p_{n}(x)$ by $f_{n}(x), \lambda_{n}$ by $i \omega_{n}$, and $\lambda_{n}^{-1} h_{n}(0)$ by $A_{n}$, formula (51) is checked. Notice that coefficient $A$ cannot be directly determined with this method.

\section{Example of the Green function}

Similarly, the Green function can be calculated by using the previous result. In order for the unknown function to satisfy the boundary conditions (54), or (2) and (3), it is convenient to define the following vectors:

$\boldsymbol{\psi}(x, t)=\left(\begin{array}{c}\partial_{t} g(x, t) \\ -c \partial_{x} g(x, t)\end{array}\right) ; \boldsymbol{\phi}_{s}(x, t)=\left(\begin{array}{c}c^{2} \delta\left(x-x_{0}\right) \delta(t) \\ 0\end{array}\right)$.

The first row of Eq. (52) is Eq. (1), while the second one comes from the definition of vector $\boldsymbol{\psi}$. Using Eq. (64), the solution is found to be: $\boldsymbol{\psi}(x, t)=\sum_{n} \boldsymbol{\psi}_{n}^{\alpha}(x) h_{n}(t)$, where

$$
\partial_{t} h_{n}-\lambda_{n} h_{n}=c^{2} \ell^{-1} p_{n}\left(x_{0}\right) \delta(t) .
$$

The initial conditions for the Green function imply $\boldsymbol{\psi}(x, t)=0$ for $t<0$, therefore $h_{n}(t)=0$ for $t<0$. Thus the solution of Eq. (68) is:

$$
h_{n}(t)=A_{n} e^{\lambda_{n} t} H(t) ; A_{n}=c^{2} \ell^{-1} p_{n}\left(x_{0}\right) .
$$

As a consequence,

$$
\left(\begin{array}{c}
\partial_{t} g(x, t) \\
-c \partial_{x} g(x, t)
\end{array}\right)=\sum_{n} A_{n}\left(\begin{array}{c}
p_{n}(x) \\
v_{n}(x)
\end{array}\right) e^{\lambda_{n} t} H(t) .
$$

Integrating the first row with respect to time leads to:

$$
g(x, t)=H(t) \sum_{n}\left[A_{n} p_{n}(x) e^{\lambda_{n} t}+A(x)\right] .
$$

Derivating this expression with respect to $x$ and using the second row of (69) leads to $\partial_{x} A(x)=0$, thus $A$ is a constant, as expected. In order to deduce the value of this constant, we need the following result:

$$
\partial_{t} g(x, 0)=p(x, 0)=c^{2} \delta\left(x-x_{0}\right)
$$

It is obtained by derivating the first row of (69) with respect to time, and the second row of (69) with respect to abscissa, leading to $p(x, 0) \delta(t)=c^{2} \delta\left(x-x_{0}\right) \delta(t)$ (remind that $\left.\partial_{t}[F(t) H(t)]=H(t) \partial_{t} F(t)+F(0) \delta(t)\right)$. The end of the calculation is done in section VIA 3, giving Eq. (49), by replacing $p(x, 0)$ by $g(x, 0)$ and taking into account that $g(0,0)=g(\ell, 0)=0$. We notice that the calculation is valid for both passive and active boundaries.

\section{EIGENMODES EXPANSION IN THE FREQUENCY DOMAIN: BIORTHOGONALITY}

Frequency domain approach is very popular in acoustics (see e.g. Ref.8 ), and leads to the use of biorthogonality (see e.g. Ref.17, p.884) of modes, except when the boundary impedances are imaginary, corresponding to non dissipative boundaries: for that case, modes are orthogonal, and the laplacian operator is self-adjoint. In this section we limit the discussion to the Green function calculation, and use successively the two above-used approaches : the second order equation, then the system of two first order equations, ignoring the constant mode. Because we are now in the Fourier domain, equations are ordinary differential equations, biorthogonality theory ensuring the completeness of the modes family. 


\section{A. Solution of the second order equation}

\section{Modal expansion}

In order to calculate the inverse FT of $G(x, \omega)$, another solution is possible: the expansion of $G(x, \omega)$ in eigenmodes. This is done for a particular case by Filippi ${ }^{1}$ (p. 58 : this author considers another type of excitation instead of the Dirac function, thus uses the Laplace Transform instead of the FT; notice that the constant mode is missing in this work). We will see how this method leads to the same poles and residues that the direct method using the closed-form expression (12). We are searching for the following expansion :

$$
G(x, \omega)=\sum_{n} G_{n}(x, \omega),
$$

where the eigenmodes $G_{n}(x, \omega)$ are solutions of:

$$
\left[\partial_{x x}^{2}+\theta_{n}^{2}(\omega) / c^{2}\right] G_{n}(x, \omega)=0,
$$

and satisfy the boundary conditions (7) and (8). The key point is that eigenmodes $G_{n}(x, \omega)$ and eigenfrequencies $\theta_{n}(\omega)$ depend on frequency $\omega$ : this is due to the boundary conditions, which are of Robin type. Solutions of Eqs. (73) can be written as follows:

$$
G_{n}(x, \omega)=\cosh \left(i \theta_{n}(\omega) x / c+\varphi_{n}(\omega)\right)
$$

where $\theta_{n}(\omega)$ and $\varphi_{n}(\omega)$ are given by the boundary conditions. Thus they satisfy:

$$
\begin{array}{r}
\theta_{n}(\omega) \tanh \varphi_{n}(\omega)=\omega / \zeta \\
\theta_{n}(\omega) \tanh \left(i \theta_{n}(\omega) \ell / c+\varphi_{n}(\omega)\right)=-\omega / \zeta_{\ell} .
\end{array}
$$

Eliminating quantity $\varphi_{n}(\omega)$, the eigenvalues are found to satisfy the following equation:

$$
\tanh \left(i \theta_{n}(\omega) \ell / c\right)\left[\theta_{n}(\omega)+\frac{\omega^{2}}{\theta_{n}(\omega) \zeta \zeta_{\ell}}\right]=-\omega\left[\frac{1}{\zeta}+\frac{1}{\zeta_{\ell}}\right] .
$$

When $\theta_{n}(\omega)$ and $\omega$ are not simultaneously zero, this equation can be rewritten as:

$$
e^{2 i \theta_{n} \ell / c}=\left[\frac{\theta_{n} \zeta-\omega}{\theta_{n} \zeta+\omega}\right]\left[\frac{\theta_{n} \zeta_{\ell}-\omega}{\theta_{n} \zeta_{\ell}+\omega}\right] .
$$

Calculation of all solutions of this equation is not necessary, only one of them being useful in the following. Operator $D=\partial_{x x}^{2}$ is formally equal to its adjoint $\bar{D}$, but the boundary conditions are different (conditions for $\bar{D}$ are complex conjugate of conditions for $D$ ). Modes of $\bar{D}$ are the complex conjugate of modes $G_{n}(x, \omega)$ (they are equal to modes $G_{n}(x, \omega)$ only if $\zeta$ and $\zeta_{\ell}$ are imaginary, because of the factor $i$ in boundary conditions (7) and (8)). Thus in general operator $D$ is not self-adjoint, and eigenmodes $G_{n}(x, \omega)$ and $\overline{G_{n}}(x, \omega)=G_{n}^{*}(x, \omega)$ are biorthogonal (see Ref. ${ }^{17}$ ). The scalar product of modes $G_{n}(x, \omega)$ with $\overline{G_{m}}(x, \omega)$ is simply given by:

$$
\int_{0}^{\ell} G_{m}(x, \omega) G_{n}(x, \omega) d x=\Gamma_{n} \delta_{n m}
$$

where

$$
\Gamma_{n}=\frac{\ell}{2}\left[1+c \frac{\sinh 2\left(i \theta_{n} \ell / c+\varphi_{n}\right)-\sinh 2 \varphi_{n}}{2 i \ell \theta_{n}}\right] .
$$

Therefore modes $G_{n}(x, \omega)$ are orthogonal (for the product (79)) and fulfill the same boundary conditions as $G(x, \omega)$, contrary to resonance modes $f_{n}(x)$ in Eq. (29). Finally the solution of Eq. (6) can be written as follows:

$$
G(x, \omega)=c^{2} \sum_{n} \frac{G_{n}(x, \omega) G_{n}\left(x_{0}, \omega\right)}{\Gamma_{n}\left(\theta_{n}^{2}(\omega)-\omega^{2}\right)} .
$$

\section{Calculation of poles and residues}

In order to calculate the inverse FT, the residue calculus will be used again. The only terms of the series contributing to poles verify:

$$
\theta_{n}(\omega)= \pm \omega
$$

Looking at Eq. (78), it can be seen that these two solutions lead to the same equation for $\omega$. Rewriting Eq. (78) by using Eqs. (75) and (76), the resonance modes frequencies are found to be solutions of Eq. (20). Solutions $\omega_{p}$ of this equation are the non zero poles of the integral in the inverse FT. Nevertheless the pole $\omega=0$ exists again, because the zero value satisfies Eq. (82), the eigenvalue $\theta_{n}(\omega)=0$ satisfying Eq. (77).

It remains to calculate the residues. Starting with the poles $\omega_{p} \neq 0$, we need to select in the series (81) the terms involving poles. For a given $\omega_{p}$, there are two terms. However it appears that modes corresponding to $\theta_{n}$ and $-\theta_{n}$ are identical. As a consequence, only one term of the series contributes to the inverse FT: it will be denoted $\theta_{p}(\omega)$. The corresponding residue is found by expanding Eq. (81) for $\omega$ close to $\omega_{p}$, as follows:

$$
G(x, \omega)=c^{2} \frac{G_{p}\left(x, \omega_{p}\right) G_{p}\left(x_{0}, \omega_{p}\right)}{\Gamma_{p}\left(2 \omega_{p}\right)\left(\omega_{p}-\omega\right)\left(1-\left[\frac{d}{d \omega} \theta_{p}(\omega)\right]_{\omega=\omega_{p}}\right)} .
$$

A similar expression can be found in Filippi,${ }^{1}$ which points out that Morse and Ingard $^{8}$ (p.559) forgot the derivative. The same error is found in Morse and Feshbach $^{17}$ (p.1347), with another error in the derivation of Eq. (77): these authors treated the problem of a string with one non-rigid (and resistive) support.

Actually the derivative of $\theta_{p}(\omega)$, denoted $\theta_{p}^{\prime}(\omega)$ can be calculated analytically, as follows. Taking the derivative of Eq. (78) with respect to $\omega$, or more conveniently, taking the logarithmic derivative of Eqs. (75) and (76), the following results are obtained: 


$$
\frac{1}{\omega_{p}}-\frac{\theta_{p}^{\prime}}{\theta_{p}}=\frac{2 \varphi_{p}^{\prime}}{\sinh 2 \varphi_{p}}=\frac{2\left(i \theta_{p}^{\prime} \ell / c+\varphi_{p}^{\prime}\right)}{\sinh 2\left(i \theta_{p} \ell / c+\varphi_{p}\right)}
$$

Thus, eliminating the derivative $\varphi_{p}^{\prime}$, writing $\theta_{p}=\omega_{p}$ and using Eq. (80), it is found after some algebra:

$$
1-\theta_{p}^{\prime}=\ell / 2 \Gamma_{p} .
$$

Finally, for $\omega$ close to $\omega_{p}$ :

$$
G(x, \omega)=-\frac{c^{2}}{\ell} \frac{G_{p}\left(x, \omega_{p}\right) G_{p}\left(x_{0}, \omega_{p}\right)}{\omega_{p}\left(\omega-\omega_{p}\right)},
$$

which is in accordance with Eq. (23).

Otherwise, for $\omega$ close to 0 , the solution $\theta(\omega)$ which is close to 0, solution of Eq. (82), satisfies the following equation, deduced from (77) :

$$
\left[1-\Theta^{2} / 3+O\left(\Theta^{4}\right)\right]\left[\Theta^{2}+\Omega^{2} /\left(\zeta \zeta_{\ell}\right)\right]=i \Omega\left[\zeta^{-1}+\zeta_{\ell}^{-1}\right]
$$

where $\Theta=\theta \ell / c$ and $\Omega=\omega \ell / c$. Therefore $\Theta^{2}$ is of order $\Omega$, and

$$
\theta^{2}=i \omega c \ell^{-1}\left[\zeta^{-1}+\zeta_{\ell}^{-1}\right]+O\left(\omega^{2}\right) .
$$

Using Eq. (81), the residue for the pole $\omega=0$ is obtained, and Eq. (25) is confirmed. We conclude that the method of the expansion in orthogonal modes in the Fourier domain leads to the same result (Eq. (26)) than the "direct" method, but the derivation is more delicate.

\section{B. System of two first-order equations}

We consider now the FT of (52):

$$
i \omega \boldsymbol{\psi}(x, \omega)=\boldsymbol{A} \boldsymbol{\psi}(x, \omega)+\boldsymbol{\phi}_{s}(x, \omega),
$$

where $\boldsymbol{\Psi}(x, \omega)=(P(x, \omega), V(x, \omega))^{T}$. An interest of the system is that the boundary conditions are independent of frequency:

$$
P(0, \omega)=-\zeta V(0, \omega) ; P(\ell, \omega)=\zeta_{\ell} V(\ell, \omega) .
$$

Eigenvalues $\lambda_{n}$ and eigenvectors $\boldsymbol{\psi}_{n}^{\alpha}$ of operator $\boldsymbol{A}$ are already known (see Eqs. (56) and (57)). The appendix shows that the adjoint operator of $\boldsymbol{A}$, is $\overline{\boldsymbol{A}}=-\boldsymbol{A}$, and gives the boundary conditions for it. This formulation differs slightly from the work, ${ }^{10,11}$ considering a different operator, but the principle is identical: we notice that these authors treat the problem for more general operators and boundary conditions. Eigenvalues and eigenvectors of $\overline{\boldsymbol{A}}$ are solutions of:

$$
\begin{aligned}
\overline{\boldsymbol{A}} \overline{\boldsymbol{\psi}_{m}^{\alpha}} & =\overline{\lambda_{m}} \overline{\boldsymbol{\psi}_{m}^{\alpha}} \\
\overline{p_{m}} & =\zeta \overline{v_{m}} \text { for } x=0 \quad \overline{p_{m}}=-\zeta_{\ell} \overline{v_{m}} \text { for } x=\ell .
\end{aligned}
$$

Thus, the adjoint eigenvalue problem to be solved is the same as the direct one, by replacing $c$ by $-c, \eta$ by $-\eta$ and $\eta_{\ell}$ by $-\eta_{\ell}$. The eigenelements are thus found to be:

$$
\overline{\psi_{m}^{\alpha}}=\left(\begin{array}{c}
\overline{p_{m}}(x) \\
\overline{v_{m}}(x)
\end{array}\right)=\left(\begin{array}{c}
\cosh \left(\overline{\lambda_{m}} x / c+\eta\right) \\
\sinh \left(\overline{\lambda_{m}} x / c+\eta\right)
\end{array}\right)
$$

where

$$
\overline{\lambda_{m}}=-\left(\eta+\eta_{\ell}+i m \pi\right) c / \ell=\lambda_{-m}
$$

Comparing with the family $\boldsymbol{\psi}_{n}^{\alpha}$ (Eq. (56)), there is a difference in sign for the second row: we notice that Rideau $^{4}$ made an error in the biorthogonal family. By construction, the biorthogonality relationship is ensured:

$$
\left(\lambda_{n}-{\overline{\lambda_{m}}}^{*}\right)<\boldsymbol{\psi}_{n}^{\alpha}, \overline{\boldsymbol{\psi}_{m}^{\alpha}}>=0 .
$$

Using Eq. (27), we remark that $\lambda_{n}={\overline{\lambda_{m}}}^{*}$ implies $m=$ $-\nu$, as defined in Eq. (28). Therefore

$$
<\boldsymbol{\psi}_{n}^{\alpha}, \overline{\boldsymbol{\psi}_{m}^{\alpha}}>=\int_{0}^{\ell}{\overline{\psi_{m}^{\alpha}}}^{T *} \boldsymbol{\psi}_{n}^{\alpha} d x=(-1)^{\mu} \ell \delta_{m,-\nu} .
$$

This latter relation enables to perform a modal decomposition on the $\left(\boldsymbol{\psi}_{n}^{\alpha}\right)$ family: but, contrarily to standard cases, the $n$-th coefficient is not given by the scalar product with $\boldsymbol{\psi}_{n}^{\alpha}$, but by the scalar product with $\overline{\psi_{-\nu_{n}}^{\alpha}}$, (up to the normalization coefficient $(-1)^{\mu} \ell$ ). Notice that if $\eta$ and $\eta_{\ell}$ are both real, $\overline{\lambda_{m}}=\lambda_{m}^{*}$, and (91) is obvious from the expressions of eigenvalues and eigenvectors (for this case, $\lambda_{n}={\overline{\lambda_{m}}}^{*}$ implies $n=m)$. For the general case, the scalar product can be written: $\left\langle\boldsymbol{\psi}_{n}^{\alpha}, \overline{\boldsymbol{\psi}_{m}^{\alpha}}\right\rangle=\int_{0}^{\ell} \cos \left[\beta_{n}(x)+\beta_{-m}(x)\right] d x=$ $(-1)^{\mu} \int_{0}^{\ell} \cos [(\nu+m) \pi x / \ell] d x$. Comparison with Eq. (A4) exhibits the difference between the two methods.

It remains to apply orthogonality to Eq. (86). We choose the case of the Green function (Eqs. (67)), with the following result:

$$
G(x, \omega)=-\frac{c^{2}}{\ell} \sum_{n} \frac{f_{n}(x) f_{x}\left(x_{0}\right)}{\omega\left(\omega-\omega_{n}\right)} .
$$

The calculation is easy, because $\omega_{n}$ does not depend on frequency. Comparison with Eq. (29) exhibits a difference in the denominator, i.e. a factor $\omega$ instead of $\omega_{n}$, and, of course, the absence of constant mode. When returning to the time domain, all the terms corresponding to $\omega=\omega_{n}$ are identical, and a constant mode is found for the pole $\omega=0$, but again it is not possible to deduce it from orthogonality relations, as in section VIB. Nevertheless, because of the independence of the boundary conditions with respect to frequency, the calculation of the residues is much easier than for the second-order equation. For the same reason, the calculation in the time domain would be possible with the same modal decomposition, and this is a major difference with the methods based upon the second-order equation.

\section{CONCLUSION}

The simple problem we have studied, which can be regarded in particular as a radiation problem, exhibits interesting properties for the resonance modes: they are 
complex-valued, and non orthogonal for the simple product (38) because of the bounded character of the considered medium, but except the constant mode, they are orthogonal for a product modified in a proper way, and are a basis for the space of solutions. Second order equations allow to find the constant mode, while first order systems of equations allow a more direct formulation of boundary impedances.

Thanks to the simplicity of the problem, the analytical treatment is possible with several methods, elucidating the relationship between them, which can be useful for more intricate problems (e.g. when damping is added to propagation, or when boundary impedances involve a mass or a spring). No approximations are needed, the results are valid whatever the value of the terminal resistances. Active boundaries can also be considered, thanks to a change in functions. We notice that an advantage of the frequency domain calculations is the possibility of the treatment of an arbitrary dependence of the boundary conditions. For a dependence $\eta(\omega)$ and $\eta_{\ell}(\omega)$, Eq. (29) remains valid by replacing $\ell$ by $\left[\ell-i c\left(\eta_{p}^{\prime}+\eta_{\ell p}^{\prime}\right)\right]$, where $\eta_{p}^{\prime}=(d \eta / d \omega)_{\omega=\omega_{p}}$, and similarly for $\eta_{\ell}$. This can be shown by generalyzing Eq. (22), or, with some algebra, using the modal expansion.

Finally, considering the problem of a stratified medium (see section II), it could be deduced in the field outside of the interval $[0, \ell]$. When terminations are passive, a result is that modes tend to infinity when $x$ tends to $\pm \infty$. An interesting study has been done in Ref.14, using biorthogonality and explaining the relation between the energy outside the interval and the terms responsible of non orthogonality in equation (39).

\section{Acknowledgements}

We would like to thank José Antunes, Patrick Ballard, Sergio Bellizzi, Michel Bruneau, Paul Filippi, Dominique Habault, Pierre-Olivier Mattei and Vincent Pagneux for very fruitful discussions.

\section{APPENDIX A: PROOF OF THE COMPLETENESS OF THE EIGENELEMENTS OF OPERATOR $A$}

The operator $\boldsymbol{A}$, defined by Eq.(53) is a differential operator defined on the energy space $H=L^{2}(0, \ell) \times L^{2}(0, \ell)$, it has a compact resolvent (cf Ref.5, p.191). Using the ordinary scalar product $\langle\boldsymbol{\psi}, \boldsymbol{\varphi}\rangle=\int_{0}^{\ell}\left[p q^{*}+v w^{*}\right] d x$, between $\boldsymbol{\psi}(x, t)=(p, v)^{T}$ and $\boldsymbol{\varphi}(x, t)=(q, w)^{T}$, the following result is obtained:

$$
\begin{aligned}
& <\boldsymbol{A} \boldsymbol{\psi}, \boldsymbol{\varphi}>+<\boldsymbol{\psi}, \boldsymbol{A} \boldsymbol{\varphi}>= \\
& \quad-c\left[v\left(q^{*}+\zeta_{\ell} w^{*}\right)\right]_{x=\ell}+c\left[v\left(q^{*}-\zeta w^{*}\right)\right]_{x=0} .
\end{aligned}
$$

It is deduced that the adjoint operator of $\boldsymbol{A}$ is $\overline{\boldsymbol{A}}=-\boldsymbol{A}$ (we denote all quantities related to the adjoint problem with an overline), and on its domain, the following ad- joint boundary conditions must be fulfilled:

$$
q(0, t)=\zeta^{*} w(0, t) \text { and } q(\ell, t)=-\zeta_{\ell}^{*} w(\ell, t) \quad \forall t
$$

(here $\zeta=\zeta^{*}$, and $\zeta_{\ell}=\zeta_{\ell}^{*}$; if $\zeta$ is infinite, the boundary conditions are $v(0, t)=0$, and $w(0, t)=0$, and similarly for boundary $x=\ell$ ). Therefore $\boldsymbol{A}$ is skew-symmetric, but not skew-adjoint, because the domains of $\boldsymbol{A}$ and $\overline{\boldsymbol{A}}$ are different, except if both $\zeta$ or $\zeta_{\ell}$ are either zero or infinite (Dirichlet or Neumann conditions); notice that for a skew-adjoint operator, the eigenvalues are purely imaginary. In order to find a new scalar product, we denote, from Eqs. (58) to (60):

$$
\boldsymbol{\psi}_{n}^{\alpha}(x)=\left(\begin{array}{cc}
e^{\alpha(x)} & e^{-\alpha(x)} \\
-e^{\alpha(x)} & e^{-\alpha(x)}
\end{array}\right)\left(\begin{array}{c}
e^{i \beta_{n}(x)} \\
e^{-i \beta_{n}(x)}
\end{array}\right) .
$$

In $H$ the standard scalar product $<\boldsymbol{\psi}_{n}^{\alpha}, \boldsymbol{\psi}_{p}^{\alpha}>_{H}=$ $\int_{0}^{\ell}\left[p_{n} p_{p}^{*}+v_{n} v_{p}^{*}\right] d x$ does not vanish for $n \neq p$, except if $\alpha(x)=0$. If we denote $\boldsymbol{\psi}_{n}^{0}(x)$ the functions corresponding to the latter case, it is possible to construct a new scalar product ensuring orthogonality, in a similar way Rideau ${ }^{4}$ did. From Eq. (62), the following hyperbolic rotation is obtained:

$$
\boldsymbol{\psi}_{n}^{0}(x)=\boldsymbol{G}_{\alpha}(x) \boldsymbol{\psi}_{n}^{\alpha}(x)
$$

We will now prove that the new product

$$
<\boldsymbol{\psi}, \boldsymbol{\varphi}>_{H}^{\alpha}=<\boldsymbol{G}_{\alpha} \boldsymbol{\psi}, \boldsymbol{G}_{\alpha} \boldsymbol{\varphi}>_{H}=\int_{0}^{\ell} \boldsymbol{\varphi}^{T *} \boldsymbol{M}_{\alpha}(x) \boldsymbol{\psi} d x
$$

where $\boldsymbol{M}_{\alpha}(x)=\boldsymbol{G}_{\alpha}^{T} \boldsymbol{G}_{\alpha}$, leads to the orthogonality of the modes. $\boldsymbol{M}_{\alpha}(x)$ is found to be equal to $\boldsymbol{G}_{2 \alpha}$. It is symmetrical and positive definite because

$$
\begin{array}{r}
\left(\|\boldsymbol{\psi}\|_{H}^{\alpha}\right)^{2}=\left(\left\|(p, v)^{T}\right\|_{H}^{\alpha}\right)^{2}= \\
\int_{0}^{\ell}\left[\cosh [2 \alpha(x)]\left(|p|^{2}+|v|^{2}\right)+2 \sinh [2 \alpha(x)] \Re e\left(p v^{*}\right)\right] d x
\end{array}
$$

can be rewritten as:

$$
\left(\|\boldsymbol{\psi}\|_{H}^{\alpha}\right)^{2}=\frac{1}{2} \int_{0}^{\ell}\left[e^{2 \alpha(x)}|p+v|^{2}+e^{-2 \alpha(x)}|p-v|^{2}\right] d x .
$$

Moreover $\alpha(x)$ is a function varying monotonously from $\eta_{r}$ to $-\eta_{\ell r}$ when $x$ increases from 0 to $\ell$, and the following bounds can be found for $\|\boldsymbol{\psi}\|_{H}^{\alpha}$ :

$$
c_{\alpha}\|\boldsymbol{\psi}\|_{H}<\|\boldsymbol{\psi}\|_{H}^{\alpha}<C_{\alpha}\|\boldsymbol{\psi}\|_{H}
$$

where $c_{\alpha}=e^{-\widetilde{\eta}}$ and $C_{\alpha}=e^{\widetilde{\eta}}$, with $\widetilde{\eta}=\sup \left[\left|\eta_{r}\right|,\left|\eta_{\ell r}\right|\right]$.

Therefore the modes $\boldsymbol{\psi}_{n}^{\alpha}$ are orthogonal for the new scalar product $\langle\boldsymbol{\psi}, \boldsymbol{\varphi}\rangle_{H}^{\alpha}$. First recall that $\left(\psi_{n}^{0}\right)_{n}$ is the family of eigenvectors of a classically skew-adjoint operator with compact resolvent, it is thus complete in $H$. Now, thanks to (A3), the two norms are equivalent on $H$, and the hyperbolic rotation shows that $\left(\boldsymbol{\psi}_{n}^{0}\right)_{n}$ and 
$\left(\boldsymbol{\psi}_{n}^{\alpha}\right)_{n}$ span the same subspace, namely the whole of $H$. This proves the completeness of $\left(\boldsymbol{\psi}_{n}^{\alpha}\right)_{n}$ in $H$.

The calculation leads to the simple result:

$<\boldsymbol{\psi}_{n}^{\alpha}, \boldsymbol{\psi}_{m}^{\alpha}>_{H}^{\alpha}=<\boldsymbol{\psi}_{n}^{0}, \boldsymbol{\psi}_{m}^{0}>_{H}=\int_{0}^{\ell} \cos \left[\beta_{n}(x)-\beta_{m}(x)\right]$

$=\int_{0}^{\ell} \cos [(n-m) \pi x / \ell] d x=\ell \delta_{n m}$.

${ }^{1}$ P. Filippi, "Acoustics of enclosures", in Acoustics: Basic Physics, Theory and Methods, edited by P. Filippi, D. Habault, J.P. Lefebvre, A. Bergassoli (Academic Press, San Diego, 1999), chap.2.

${ }^{2}$ G.Oliveto and A. Santini, Complex modal analysis of a continuous model with radiation damping, J.Sound Viv. 192, 15-33 (1996)

${ }^{3}$ J.L.Guyader, Vibrations des milieux continus (Vibration of continuous media) (Lavoisier, Hermès, Paris, 2002)

${ }^{4} \mathrm{P}$. Rideau, Contrôle par assemblage de poutres flexibles par des capteurs-actionneurs ponctuels: étude du spectre du système (Control by assembling of flexible beams using discrete sensorsactuators: study of the spectrum of the system), PhD Thesis, Ecole Nationale Supérieure des Mines de Paris (1985)

${ }^{5} \mathrm{~A}$. Intissar, Analyse Fonctionnelle et théorie spectrale pour les opérateurs compacts non auto-adjoints (Functional analysis and spectral theory for compact, non self-adjoint operators), (Cepadues, Toulouse, 1997)

${ }^{6} \mathrm{~S}$. Cox and E. Zuazua, The rate at which energy decays in a string damped at one end, Indiana University Mathematics Journal, 44, 545-573,(1995).

${ }^{7}$ Darmawijoyo and W.T. Van Horssen, On the weakly damped vibrations of a string attached to a spring mass dashpot system, Journal of Vibration and Control 9, 1231-1248 (2003)

${ }^{8}$ P. M. Morse and K.U. Ingard, Theoretical Acoustics (Mc Graw Hill, 1968)

${ }^{9}$ M. Ochmann, U. Donner, Investigations of silencers with asymmetrical lining. I: Theory, Acta Acustica 2 , 247-255 (1994)

${ }^{10} \mathrm{R}$. Rabenstein and L. Trautmann, Multidimensional transfer function models, IEEE Transactions on circuits and systems 49, 852-861, 2002.
${ }^{11}$ L. Trautmann and R. Rabenstein, Digital sound synthesis by physical modeling using the functional transformation method, Kluwer, New York, 2003.

${ }^{12}$ H. Levine, Acoustical cavity excitation, J. Acoust.Soc.Am.109, 2555-2565 (2001).

${ }^{13}$ P.T.Leung, S.Y.Liu, K.Young, Completeness and orthogonality of quasinormal modes in leaky optical cavities, Phys.Rev.A, 49, 3057-3067, 1994

A4) ${ }^{14}$ P.T.Leung, W.M. Suen, C.P.Sun, K.Young, Waves in open systems via a biorthogonal basis, Phys.Rev.E, 57, 6101-6104, 1998

${ }^{15} \mathrm{~J}$. Kergomard, Elementary Considerations on Reed-instruments Oscillations, in Mechanics of Musical Instruments, edited by A. Hirschberg, J.Kergomard, G. Weinreich (Springer-Verlag, Wien, 1995)

${ }^{16}$ V.Debut, Deux études d'un instrument de musique de type clarinette: analyse des fréquences propres du résonateur et calcul des auto-oscillations par décomposition modale (Two studies of a clarinetlike musical instrument: analysis of the resonator eigenfrequencies and calculation of self-sustained oscillations using modal expansion), PhD Thesis, Université Aix-Marseille II, 2004.

${ }^{17}$ P.M. Morse and H. Feshbach, Methods of theoretical physics (Mc Graw Hill, 1953)

${ }^{18} \mathrm{~J}$.Woodhouse, Idealized models of a bowed string, Acustica, 79, 233-250 (1993)

${ }^{19}$ K.M.Ahmida and J.R.F. Arruda, On the relation between complex modes and wave propagation phenomena, J. Sound Vib. 255, 663-684, (2002)

${ }^{20}$ L. Meirovitch, Elements of vibration analysis (Mc Graw Hill 1975)

${ }^{21}$ D.L.Russell, Control theory of hyperbolic equations related to certain questions in harmonic analysis and spectral theory, J.Math.Anal.Appl.40, 336-368 (1972)

${ }^{22}$ A.Majda, The location of the spectrum for the dissipative acoustic operator, Indiana Univ. Math. J. Journal 25, 973-987 (1976)

${ }^{23}$ J.Lagnese, Decay solutions of wave equations in a bounded region with boundary dissipation, J. Differential Equations, 50, 163-182 (1983)

${ }^{24}$ H.T. Banks, G. Propst and R.J.Silcox, A comparison of time domain boundary conditions for acoustic waves in wave guides, Quart. Appl. Math. 54, 249-265 (1996) 


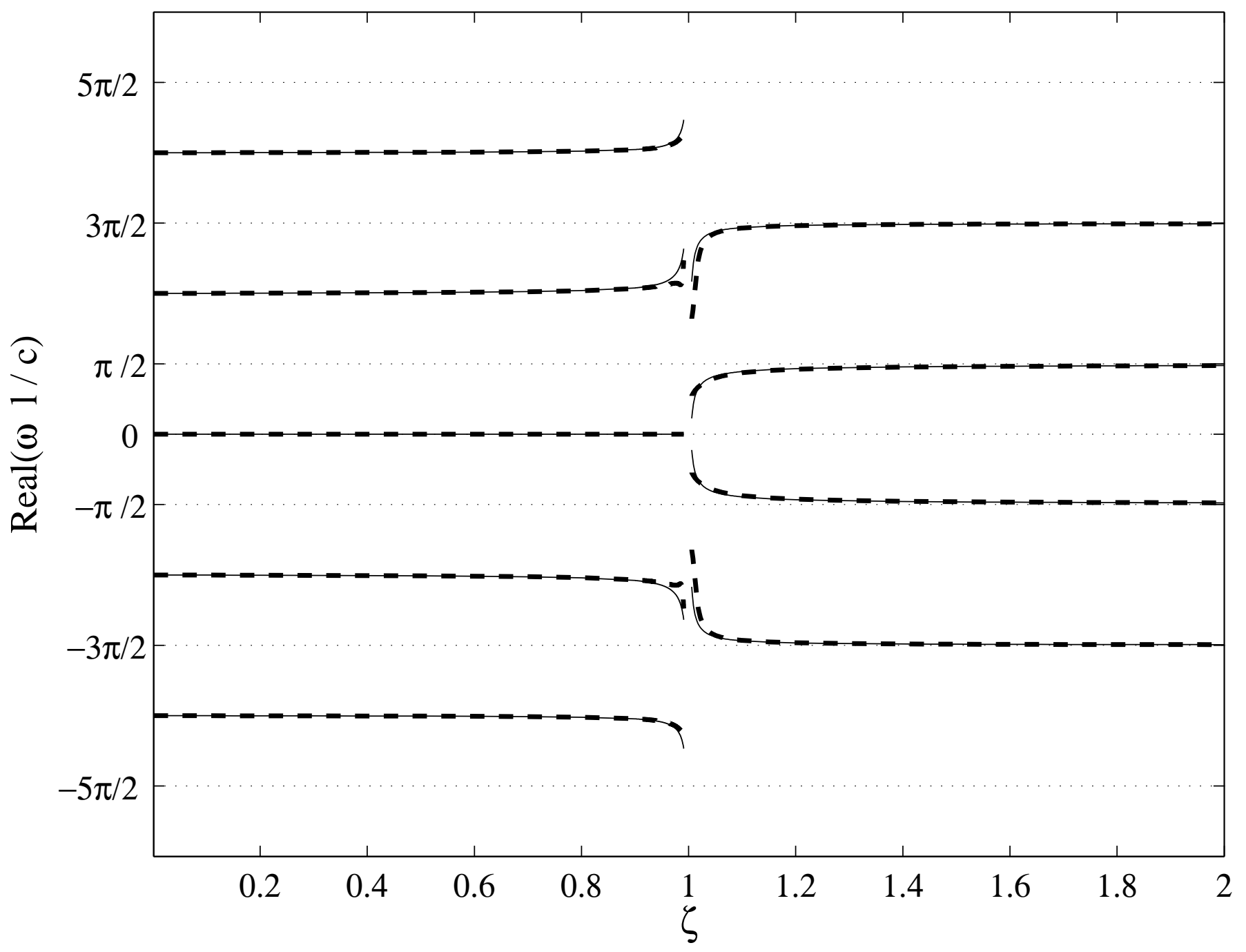




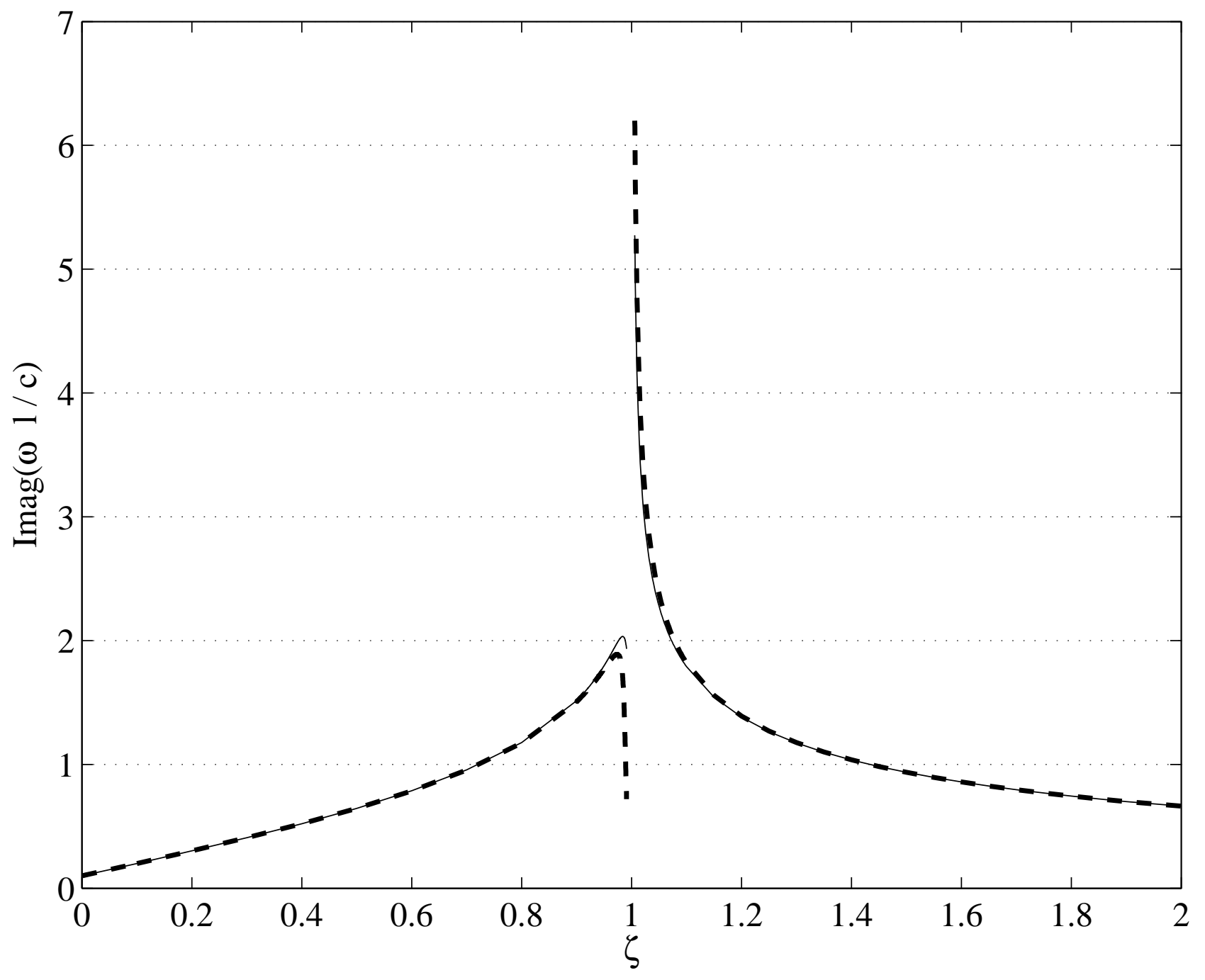




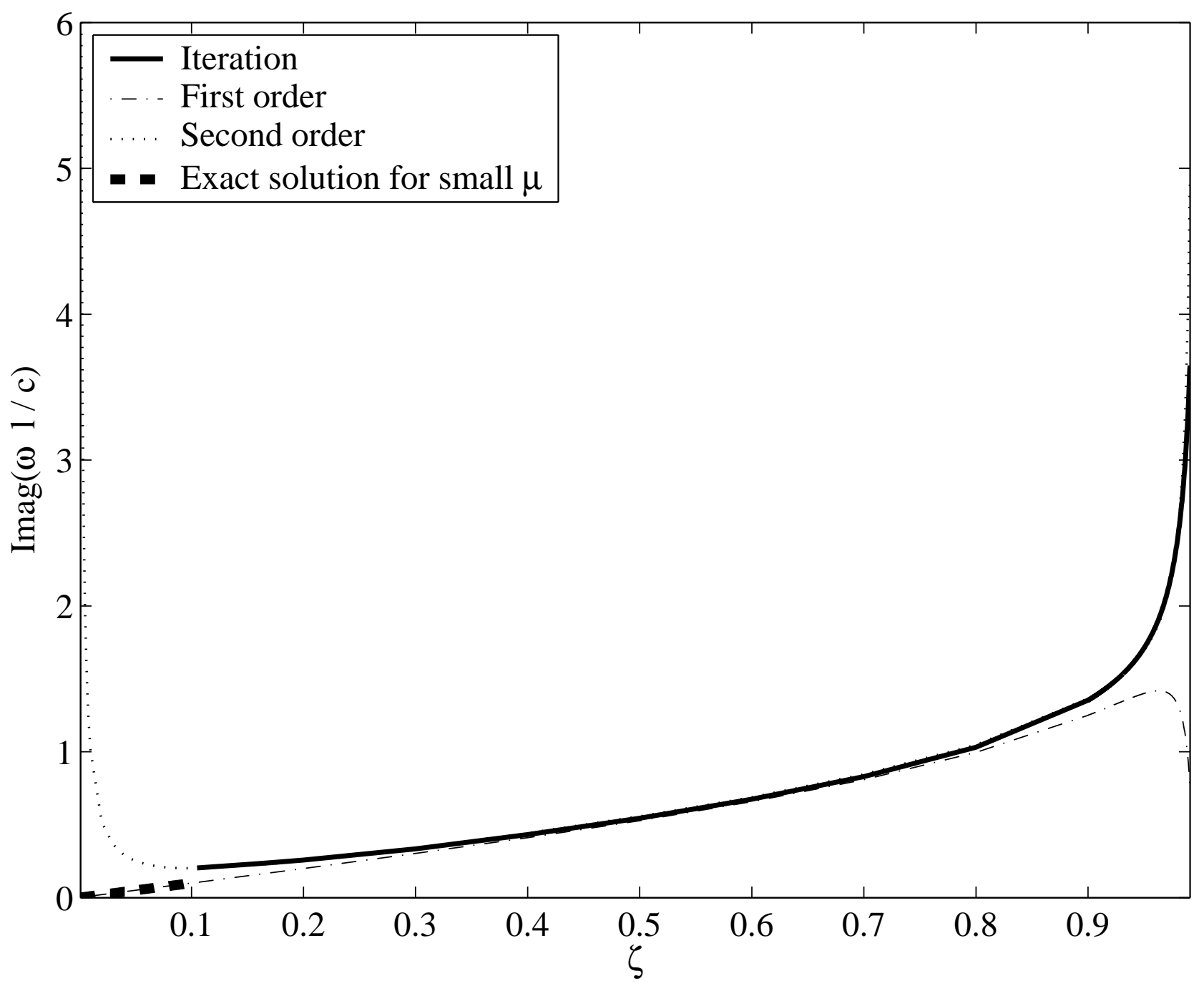




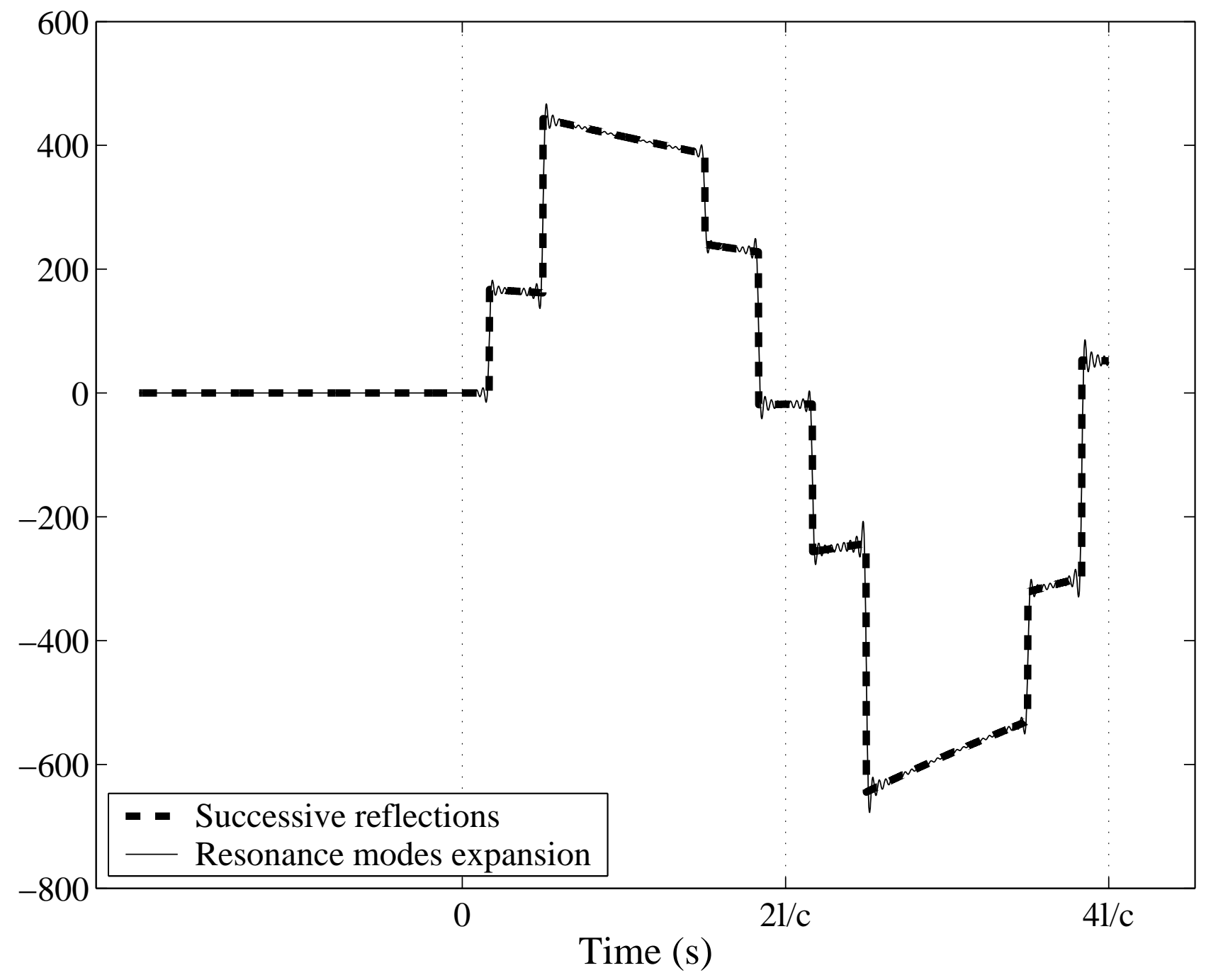




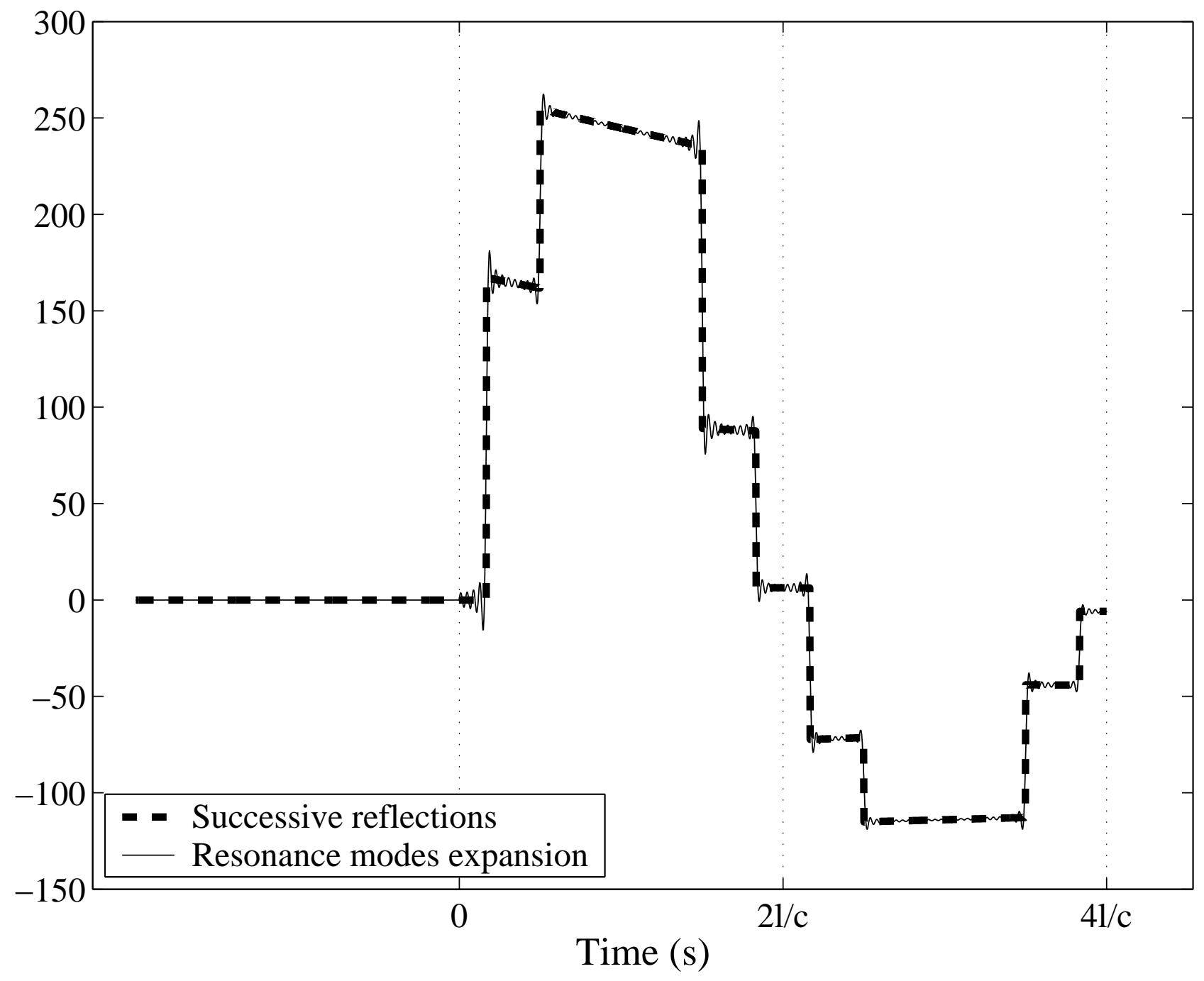

